\title{
New Frontiers in Cancer Therapy: Monoclonal Antibody Therapy of Hematologic Malignancies
}

\author{
Reviewed by Akifumi Takaori-Kondo \\ Department of Hematology/Oncology, Graduate School of Medicine, \\ Kyoto University, Kyoto, Japan
}

Int J Hematol. 2002;75:548.

(C)2002 The Japanese Society of Hematology

New Frontiers in Cancer Therapy: Monoclonal Antibody Therapy of Hematologic Malignancies, edited by Bruce D. Cheson. Oxfordshire, United Kingdom: Darwin Scientific Publishing, 2001; 287 pages.

This book begins with a chapter on historical aspects of the development of antibody therapy. In this chapter, the author starts by quoting the term "magic bullet," used by Paul Ehrlich for antibody therapy, and introduces a story about Ehrlich and von Behring. This magic bullet became real with the development by Köhler and Milstein of the hybridoma technique for production of monoclonal antibodies (MoAbs). And now, 25 years later, these magic bullets are in clinical use. This chapter is the most interesting part of this book as a good literary work.

This book contains another 14 chapters: 1 on mechanisms of action and resistance, 4 on rituximab, 1 on CAMPATH$1 \mathrm{H}, 1$ on immunoconjugates, 3 on radioconjugates, and so on. This ratio of chapters per topic seems to reflect the information available about these reagents.

In the chapter on mechanisms of action and resistance, both are discussed in detail from many aspects including the antibodies themselves, antigens, immune effector mechanisms such as antibody-dependent cell-mediated cytotoxicity (ADCC) and complement-dependent cytotoxicity (CDC), and direct antiproliferative effects. This chapter is very instructive and easy to understand.

The next 4 chapters are written about rituximab and its use in the treatment of 4 different clinical disease entities: indolent lymphoma, diffuse large B-cell and mantle cell lymphomas, chronic lymphocytic leukemia, and other CD20- positive hematologic malignancies. Although these chapters overlap on some topics, such as introductory information on this MoAb, these sections are generally very rich in content, reflecting the fact that this MoAb was the first approved for clinical use and is prototypic for many other antibodies coming in the future. Many investigators and groups intensively conducted many clinical trials, which showed that rituximab was very well tolerated and effective for many types of diseases expressing CD20 antigen. However, as any physician would feel, there seems to have been much enthusiasm as well as much confusion among doctors who began treating patients with this reagent. Of course, enthusiasm is based on the evidence of the effectiveness of this reagent, whereas confusion comes from the question of how we should use this reagent appropriately. These chapters are well written in this aspect, describing the results of many clinical trials. These trial results give us many suggestions about guidelines for the use of rituximab, although these guidelines are not yet conclusive.

The chapters on radioconjugates are also rich in content, especially the chapter about iodine131 tositumomab (Bexxar). In that chapter, 2 completely different uses of this reagent are discussed, one in nonmyeloablative doses in clinical trials and another in the setting of autologous stem cell transplantation.

The last chapter, written by the editor himself, is a good summary of the whole book. This book begins with a literary article, followed by very rich contents about many reagents for various diseases, and ends with a very good summary. I think this is the book everyone should have who wants to know about the MoAb therapy of hematologic malignancies. Highly recommended. 